\title{
Poussières et fibres en tant que nuisances de l'environnement professionnel - Rôle de l'OIT
}

\author{
M. Mokrane \\ Service de la sécurité et de l'hygiène du travail, Bureau international du Travail, Genève
}

1. Depuis sa création en 1919, l'Organisation internationale du Travail, seule organisation tripartite, a mobilisé ses moyens d'action pour accomplir

sa mission d'amélioration des conditions et du milieu de travail, qu'il s'agisse de normes internationales, de réunions d'experts, d'études et de recherches, de diffusion de l'information ou encore d'activités pratiques. En dépit des progrès réalisês, la lutte contre les risques liés au milieu de travail demeurent un objectif prioritaire dont la réalisation est assurée par une contribution importante de l'hygiène industrielle qui identifie, évalue et contrôle tous les polluants chimiques ( $80 \%$ des risques) physiques et biologiques ( $20 \%$ des risques) présents sur les lieux de travail. Les poussières et fibres en tant que nuisances de l'environnement professionnel, continuent de poser l'un des problèmes les plus ardus auxquels l'industrie doit faire face dans le domaine de l'hygiène du travail tant du point de vue médical que de la prévention et du traitement.

2. La lutte contre l'exposition professionnelle aux poussières, s'est notamment traduite par la publication: 2.1. Du rapport international sur la prévention et la suppression des poussières dans les mines, les galeries et les carrières, 1983 . Ce rapport périodique récapitule les questions liées à la prévention et à la suppression des poussières, à partir d'informations fournies par les Etats Membres.

2.2. Du guide pour la prévention et la suppression des poussières dans les mines, les galeries et les carrières, 1965. Une révision de ce guide technique est en préparation. Il existe aussi un règlement type de sécurité pour les travaux souterrains dans les mines de charbon, à l'usage des gouvernements et de l'industrie charbonnière, 1950. Un projet révisé sera publié en 1985.

2.3. Des épreuves fonctionnelles respiratojres dans les pneumoconioses, 1965. Ce rapport fait le bilan de l'expérience acquise dans l'utilisation des tests de la fonction respiratoire dans les pneumoconioses. Sa révision est envisagée.

2.4. Des instructions pour l'utilisation de la Classification internationale du BIT des radiographies de preumoconioses, 1980. Elles visent à normaliser les méthodes de classification des radiographies de pneumoconioses et à permettre une comparaison au plan international, des statistiques de pneumoconioses et des résultats de recherche.

2.5. La Conférence internationale sur les pneumoconioses. Elle se tient tous les trois ans et permet, dans un cadre multidisciplinaire, l'examen de tous les aspects scientifiques des problèmes des pneumoconioses. Elle favorise une meilleure information réciproque, une confrontation d'expériences et une coopération étroite des chercheurs du monde entier. Des groupes de travail sont constitués pour examiner des questions spécifiques comme la classification internationale des radiographies, les épreuves fonctionnelles respiratoires, les risques liés à l'utilisation de l'amiante, etc.

3. Le BIT a déployé diverses activités dans la lutte contre l'exposition professionnelle à l'amiante.
3.1. Le recueil de directives pratiques pour la sécurité dans l'utilisation de l'aniante, 1984. Il établit les principes susceptibles d'être appliqués pour le contrôle des dangers spécifiques et prévoir les dispositions relatives à la sécurité et à la santé des travailleurs dans l'utilisation de l'amiante.

3.2. Le rapport VI (1) "La sécurité dans l'utilisation de l'amiante", 1984. Ce rapport (Conférence internationale du Travail, juin 1985) fait le point sur les effets de l'exposition à l'amiante, sur les mesures préventives et sur les activités pertinentes de l'OIT et autres organisations. Il contient un questionnaire auquel les gouvernements sont invités à donner des réponses motivées, sur la base desquelles le Bureau préparera un rapport sur les instruments internationaux souhaités par les Etats Membres.

4. Certains instruments internationaux touchent directement ou indirectement à ces questions.

4.1. La nouvelle liste des maladies professionnelles annexée à la Convention (no 121) sur les prestations en cas d'accidents du travail et des maladies professionnelles, 1964 où figurent le cancer pulmonaire et le mésothéliome causés par l'amiante, l'asbestose ayant été incluse dans la liste de 1964.

4.2. La Convention (no 139) et la Recommandation (no 147) sur le cancer professionnel, 1974, renferment un certain nombre de principes d'organisation de la prévention technique et médicale des risques professionnels causés par les substances et agents cancérogènes.

4.3. La Convention (no 148) et la Recommandation (no 156) sur le milieu de travail (pollution de l'air, bruit et vibrations), 1977 énonce certains principes : prescrire les mesures à prendre sur les lieux de travail pour prévenir les risques considérés, les limiter et protéger les travailleurs; fixer des critères permettant de définir les risques d'exposition et, le cas échéant, préciser les limites d'exposition qui seront révisées périodiquement; fixer les circonstances et les modalités de la surveillance médicale et spécifier les procédés, les substances, les machines ou les matériels dont l'utilisation doit être notifiée sinon autorisée ou interdite; agir en consultation étroite avec les organisations les plus représentatives des employeurs et des travailleurs intéressées.

5. Pour faciliter l'échange d'information, le BIT a mis en place :

5.1. le Système international pour la sécurité et la santé des travailleurs qui peut, grâce à un réseau mondial de 100 organismes désignés, diffuser rapidement des informations scientifiques et techniques sur de nouveaux risques et les méthodes de prévention adéquates. 5.2. Le Centre international d'information de sécurité et d'hygiène du travail (CIS)assisté par près de 50 Centres nationaux, assure sa mission d'information principalement par la publication d'analyses bibliographiques des documents les plus importants parus dans le monde, sur les aspects de sécurité et d'hygiène du travail. 6. Dans le cadre des activités de coopération technique, le BIT aide à la mise en place et/ou au renforcement d'institutions scientifiques et techniques, contribuant notamment à améliorer l'hygiène du travail. 